\title{
Discourse relations: Genre-specific degrees of overtness in argumentative and narrative discourse
}

\author{
Carolin Hofmockel *, Anita Fetzer and Robert M. Maier \\ Applied Linguistics (English), University of Augsburg, Universitaetsstrasse 10, 86159 Augsburg, \\ Germany
}

\begin{abstract}
Based on a contrastive approach that compares the argumentative genre of editorial with the narrative genre of personal narrative across two production modes, single-authored data and data elicited in an editing-based task, this paper examines the impact of genre on the realizations of discourse relations, paying particular attention to the argumentative genre and its preferential realizations of Contrast, Continuation, Elaboration and Explanation/Result. Discourse relations are conceived of as sociocognitive constructs that are encoded in coherence strands and may additionally be signalled overtly with discourse connectives, metacomments and/or pragmatic word order. Quantitative analyses of single-authored and co-constructed data reveal systematic differences in the variation of degrees of overtness between editorials and personal narratives. In the argumentative genre, Explanation, Continuation and Comment tend to be realized with a lower degree of overtness, Elaboration with a higher degree of overtness. Contrast is realized overtly throughout the data, irrespective of genre.
\end{abstract}

Keywords: Discourse relation, degree of overtness, discourse connective, genre, adjacency, production format, editing-based task

\section{Introduction}

Argumentation can be described as a language game, i.e. a social action in and through which controversial standpoints are negotiated. Dialectical approaches to argumentation model argumentation structure to reflect the process of this negotiation, with structural units representing argumentative moves that support or attack a standpoint (e.g. [12,26]) and that may be signalled with linguistic indicators [36] such as therefore and although. Recent studies in Argument Mining, aiming for the automatic detection and annotation of argumentation and its constitutive units in naturally occurring language data, have pointed out correspondences between argumentation structure, conceptualized in terms of argumentative moves, and discourse structure, conceptualized in terms of discourse relations (e.g. $[2,22,30]),{ }^{1}$ and argue for a combination of Argumentation Theory with approaches to discourse structure to develop more effective methods of automatic annotation (e.g. [6,28]).

From a discourse perspective, the structure of the discursive realization of argumentation is considered as a sequence of discourse units which are connected with semantics-based discourse relations such as Contrast, Elaboration or Comment. With regard to their linguistic realization, discourse relations may be

\footnotetext{
${ }^{*}$ Corresponding author. Tel.: +49(0)821-598-5754; E-mail: carolin.hofmockel@ philhist.uni-augsburg.de.

${ }^{1}$ In this paper, "discourse relation" is used as an umbrella term comprising discourse relation [2], rhetorical relation [22] and coherence relation $[29,30]$, to name the most common terms.
} 
encoded in coherence strands [13], and additionally may be signalled overtly, for instance with discourse connectives or pragmatic word order [21]. In the context of argumentative discourse, semantics-based discourse relations may fulfil particular argumentative functions at particular stages in the discourse. For example, discourse units realizing Continuation may fulfil the argumentative function of Support, and discourse units realizing Contrast may fulfil the function of Rebuttal; this is because discourse structure may coincide with argumentative structure. Such structural coincidences suggest that overt signals of discourse relations may be operationalizable for the automatic annotation of both argumentation structure and discourse structure.

Recent studies of the linguistic realization of discourse relations (e.g. [21,33]) indicate that the overt vs. implicit realization of DRs varies systematically according to their semantics, to the adjacent or nonadjacent positioning of discourse units realizing discourse relations, and to the contextual constraints and requirements of genre. Since overt signals of discourse relations typically serve as cues in discourse structure annotation tools, the hypothesis of genre having an influence on the linguistic realization of discourse relations may carry considerable implications for automatic discourse structure annotation, and for approaches in Argument Mining that plan to exploit discourse structure annotation. The assignment of discourse relations on the basis of overt signals may be refinable by an explicit accommodation of genre-preferential realizations: automatic annotation should thus be not only context- but also genresensitive.

Focussing on the preferences of argumentative discourse in the realization of the discourse relations Continuation, Contrast, Elaboration and Explanation/Result, which are seen as important relations for the realization of argumentation (cf. [34]), the present paper aims to provide new insights to the impact of genre on the overt vs. implicit linguistic realizations of discourse relations. It employs a doubly contrastive approach that compares the argumentative genre of editorial with the narrative genre of personal narrative both within and across two production modes: single-authored texts from British media discourse and student essays, and co-constructed texts elicited in an editing-based task specifically designed for evaluation of genre-preferential realization patterns. Object of comparison in the contrastive analyses is the degree of overtness, i.e. the proportion of overt realizations among all realizations of a given type of discourse relation or of all discourse relations in a dataset.

The paper is structured as follows. Section 2 discusses definitions and linguistic realizations of discourse relations, while Section 3 investigates the constraints and requirements of argumentative discourse and considers possible correspondences between argumentation structure and discourse structure. Sections 4 and 5 present the empirical study, introducing data, methods, results, and their analysis. Section 6 discusses the results of the contrastive analysis with a focus on argumentative discourse, while Section 7 summarizes and concludes.

\section{Discourse structure}

The analysis of discourse structure concentrates on the sequential organization of discourse units (DUs), investigating hierarchies and the semantics and pragmatics of the connectedness between constitutive parts of discourse and the discourse-as-a-whole. The nature of the connectedness of DUs clause-based in our study - is captured with the concept of discourse relations (DRs), whose overt and implicit realization is the object of investigation of this study.

In line with Thibault [35] and Levinson [19] we assume that discourse is constrained by the delimiting frame of discourse genre, or activity type. What is more, Thibault [35] introduces another important differentiation to the analysis of discourse, the distinction between type and token: 
genres are types. But they are types in a rather peculiar way. Genres do not specify the lexicogrammatical resources of word, phrase, clause, and so on. Instead, they specify the typical [original emphasis] ways in which these are combined and deployed so as to enact the typical semiotic action formations of a given community. ([35], p. 44)

In other words, discourse genre is a delimiting frame of reference with genre-specific constraints for preferred (or: default) configurations and linguistic realizations, holding for the macro unit of genre as well as for its constitutive micro units. And it is these micro units - DUs and the linguistic realization of DRs connecting them - which may be realized with different tokens. 'Typical ways' of doing things with words in discourse imply that language users can act in accordance or disaccordance with those typical ways. Acting in disaccordance with a default generally functions as an inference trigger - as is the case with flouting one or more of the Gricean maxims - and getting in a conversational implicature to calculate the speaker-intended meaning.

Levinson's [19] concept of activity type highlights the meaning-making mechanisms anchored to genre-specific constraints, holding for both the activity-type-as-a-whole as well as for its constitutive parts and the nature of their connectedness:

There is another important and related fact, in many ways the mirror image of the constraints on contributions, namely the fact that for each and every clearly demarcated activity there is a set of inferential schemata [original emphasis]. These schemata are tied to (derived from, if one likes) the structural properties of the activity in question. ([19], p. 370).

The delimiting frame of discourse genre thus provides defaults for linearization and lexical representation on various levels of analysis. The level this study concentrates on is the overt and implicit realization of DRs.

\subsection{Discourse relations}

In line with SDRT [2] a DR is defined as a logical connection between a proposition $p^{1}$ as part of a discourse $\mathrm{D}$ and some other proposition $p^{2}$ in $\mathrm{D} ; p^{1}$ and $p^{2}$ may be realized through adjacently or nonadjacently positioned DUs. A DR is thus a function that takes the two propositions under consideration as its arguments. To study the linguistic realization of DRs in naturally occurring discourse, the SDRTbased definition has been supplemented [21] by the functional-grammar concept of coherence strands [13] and the syntactic concept of theme zone $[15,16]$. On the most fine-grained level of analysis, DUs which realize propositions that are linked through a DR are referred to as elementary DUs. These typically take the form of clauses, but may also be realized as subclausal (or: "clause-like" [23]) units, such as complex noun phrases. Elementary DUs may combine to realize DRs on coarser levels, with DRs spanning across several clauses or paragraphs, which in themselves form more complex propositions.

DRs in SDRT are classified according to their semantics, their "defining conditions" in our terms. SDRT's taxonomy is thus not as fine-grained as that of Rhetorical Structure Theory (RST) [22], which, for example, "distinguishes among several Contrast relations, for instance contrast, antithesis and concession. These relations differ on the basis of quite subtle differences in their underlying semantic dissimilarity, but not with respect to the communicative intentions realized" ([2], p. 145). Table 1 systematizes the most frequent DRs with respect to their defining conditions and illustrates how DRs in SDRT categorize into coordinating and sub-/superordinating, depending on whether DUs keep the discourse on the same level, or are located on different hierarchical levels. 
Table 1

DRs and their defining conditions

\begin{tabular}{|c|c|}
\hline $\mathrm{DR}$ & Defining conditions \\
\hline \multicolumn{2}{|l|}{ Coordinating } \\
\hline Continuation & Common topic \\
\hline Narration & $\begin{array}{l}\text { Common topic } \\
\text { Temporal sequentiality }\end{array}$ \\
\hline Contrast & Semantic dissimilarity between $p^{2}$ and $p^{1}$ \\
\hline Background & $\begin{array}{l}p^{2} \text { forms the background of } p^{1} \\
\text { Common topic }\end{array}$ \\
\hline \multicolumn{2}{|c|}{ Subordinating/Superordinating } \\
\hline Result & $\begin{array}{l}p^{1} \text { gives reason for (parts of) events in } p^{2} \\
\text { Connecting two sub-events } \\
\text { Temporal precedence of cause }\end{array}$ \\
\hline Comment & $p^{2}$ selects $p^{1}$ as topic; or: $p^{1}$ selects $p^{2}$ as topic \\
\hline Elaboration & Topic of $p^{2}$ specifies topic of $p^{1}$ mereologically \\
\hline Explanation & $\begin{array}{l}p^{2} \text { gives reason for (parts of) events in } p^{1} \\
\text { Temporal consequence }\end{array}$ \\
\hline
\end{tabular}

\subsection{Linguistic realization}

Linguistically, DRs may be realized implicitly or overtly, depending on whether the defining conditions of a DR are encoded in coherence strands, or additionally signalled with discourse connectives (DCs), metacomments and/or pragmatic word order [21]. Coherence strands, in line with Givón [13], hold across clauses and are made manifest through topic continuity, i.e. topic identity or specification, tense and aspectual coherence (including modality), spatial coherence and lexical coherence, in particular lexical relations such as antonymy, synonymy etc.

Among overt signals, pragmatic word order refers to non-congruently configurated theme zones, which are adopted from Systemic Functional Grammar, in particular multiple themes and theme zone $[9,15,16]$. They realize both anaphoric and cataphoric reference by connecting what has just been said/written with what is going to be said/written. Similarly, DCs function relationally in that they index metacommunicative information about the speaker's attitude towards a DU or how DUs are to be related. They fulfil discourse-management functions and provide instructions for discourse processing and construal of discourse coherence. They may trigger generalized conversational implicatures for the structuring of discourse, and for attitude specification towards propositions and participants.

As an example, Contrast in (1) is realized overtly. Its defining condition is encoded in topic and referential continuity ('London' - 'it'), temporal discontinuity ('was' - 'is') and lexical antonymy ('dowdy'; 'stale' - 'exciting'); a contrastive DC 'but' and pragmatic word order (fronted temporal adjuncts 'in the 1950s' and 'today') additionally signal the defining condition overtly. ${ }^{2}$ In (2), Explanation is realized implicitly; it is encoded in topic continuity, temporal-aspectual overlap and the lexeme 'cause'.

(1) \#1/2 [\{In the 1950s, $\}$ London was a DOWDY place of tea-houses and STALE rock cakes.] \#1/3 [\{But today, $\}$ it's MUCH MORE EXCITING.]

(2) \#1/12a [English has gradually become the LINGUA FRANCA,]

\footnotetext{
${ }^{2}$ In this and all following examples, square brackets ('[ ]') indicate granularity, curly brackets (' \{\} ') mark material added in editing-based tasks, boldface indicates overt signals of DRs, underlining indicates referential and/or topical coherence, italics indicate temporal and aspectual coherence, and SMALL CAPS mark lexical coherence and indices.
} 


\section{\#1/12b [CAUSING interest in FOREIGN LANGUAGE to wane.]}

As regards their linguistic realization, DRs may be fully specified by indexical reference to all of their defining condition(s); they may even be overspecified if an overt signal is added to their full specification. Since some of the defining conditions of DRs show some overlap (e.g. Continuation and Narration share the defining condition of common topic, but differ in the Narration-defining condition of temporal sequentiality; cf. Table 1), their linguistic realization may also remain underspecified and thus ambiguous unless supplemented by overt signals, which guide the recipients in their assignment of the speaker/writer-intended DR. Depending on the number of conditions and features indexed in discourse, we may thus distinguish between various degrees of specification ${ }^{3}$ and various degrees of overtness.

\subsection{Variation}

Research into the linguistic realization of DRs shows that the variation between overt and implicit realizations of DRs is systematic, and appears to be affected by three variables: (1) the semantics of the DR, (2) the adjacent or non-adjacent positioning of the DUs realizing the relation, and (3) the contextual constraints and requirements of genre. Studies on the linguistic realization of DRs in English and German editorials and narratives [32,33], for instance, show that the DR Contrast is always realized overtly, indicating that overt realization is the default for this DR; other DRs such as Explanation are realized overtly in narratives more frequently than in editorials, showing systematic patterns of variation that also correlate with the sequential realization of DRs through adjacently or non-adjacently positioned DUs, with narratives realizing DRs connecting non-adjacently positioned DUs overtly more frequently than editorials. Similar tendencies are observed by [17,21] and [11] in texts elicited in editing-based tasks where participants were asked to "flesh out" bare texts of the respective genres, suggesting that discourse genre constrains the linguistic realization of DRs.

Genre-specific DR realization would mean that there are some discourse genres in which adjacently positioned Elaborations and Continuations are typically signalled overtly, and other discourse genres in which adjacently positioned Elaborations and Continuations are typically encoded, but not additionally signalled; but there is also discourse in which the realizations of the DRs are not in accordance with the typical preferences of the genre. This systematic variation is assumed to manifest itself in varying degrees of overtness of a given type of DR and of all DRs in a genre. The following section will focus on argumentative discourse and correspondences between argumentation structure and discourse structure to prepare the ground for a study of genre-preferential degrees of overtness in the linguistic realization of DRs in the specific context of argumentative discourse.

\section{Argumentative discourse and discourse structure}

\subsection{Argumentative discourse}

Argumentative discourse refers to the discursive realization of argumentation, i.e. discourse with the communicative purpose to negotiate the validity of a standpoint at issue. To achieve this goal, interlocutors may need to provide further evidence to rebut doubts and persuade their communication partner to

\footnotetext{
${ }^{3}$ Specification is seen as a purely structural phenomenon, which contributes to varying degrees of overtness, depending on the semantics of a DR, its sequential status and genre. With [21], we assume that underspecification of DRs at particular stages in discourse can be accounted for in terms of cognitive economy, and that salience may account for their overspecification.
} 
adopt their standpoint. Structural approaches to argumentation, such as Argument Mining, aim to analyse the structure of argumentative discourse by identifying and segmenting argumentative portions of discourse, and visualizing argumentation structure in diagrams. Annotation schemes, or diagramming techniques, used for these tasks differ depending on underlying frameworks.

While traditional approaches to argumentation structure concentrate mainly on the structural representation of individual arguments put forward in argumentative discourse, which they consider to be made up of a conclusion and one or more premises, dialectical approaches (e.g. [12,26]) strive for a model of how premises and conclusions and the arguments they form combine in larger complexes in an argumentation, i.e. the "macrostructure of argumentation" [12]. They consider argumentation structure as a reflection of the hypothetical exchange between a proponent who presents and supports arguments and an opponent who attacks the proponent's arguments and their supporting premises. Accordingly, text is segmented in such a way that units of analysis reflect the moves of proponent and of opponent.

Depending on whether argumentative moves defend the proponent's standpoint or attack it, they classify as Support or Attack. In line with Peldszus and Stede [26], Support moves may directly support a conclusion, realized in a move referred to as Claim, in the form of independent arguments, or they may indirectly support a conclusion by providing a new argument for, or an example in support of, one of its premises. Attack moves directly provide arguments against a conclusion or attack the cogency of a supporting argument, thus indirectly attacking a conclusion. They comprise Rebuttals against the conclusion or a supporting premise, i.e. moves that support the refutation of the conclusion or of one of the premises, and Undercuts, viz. moves that are intended to question the validity of a premise. Counter-attacks, as their name implies, are proponent's moves that attack the opponent's attacks.

According to van Eemeren et al. [36], argumentative moves may be signalled by linguistic indicators, viz. "verbal means arguers use to indicate the functions of the various moves that are made in an argumentative discussion or text" ([36], p. 479). These include DCs like but, although and nevertheless, lexical expressions such as cause, effect and leads to, and metacomments like it has not been proven that; they are considered to be operational in the automatic annotation of argumentative moves [8]. The notion of linguistic indicators ties in with other approaches in Argumentation Theory that conceive of argumentative DCs such as but, however, as a consequence as "argumentative connectors" [1], "metalinguistic operators" [25] or "pragmatic connectives" [24] which constrain the argumentative potential of the premises they introduce. Moreover, the idea of metacomments as linguistic indicators is mirrored in "shells" [20], that is high-level organizational elements such as the point is that or the argument states that, which interlocutors may use to explicitly refer to their own or an opponent's premises and conclusions. As with the overt signalling of DRs, not every move is signalled by a linguistic indicator [36], and a context-independent one-to-one mapping between linguistic indicators and argumentative moves is not feasible.

Linguistic indicators for argumentation and overt DRs signals share similar forms and functions in that both may comprise DCs and metacomments, and in that they overtly signal the connectedness of the units pertaining to their respective level of discourse representation. The following section will explore whether and in what way argumentation structure may correspond with discourse structure.

\subsection{Argumentation structure and discourse structure}

Argument Mining has investigated possible correspondences between argumentation structure and discourse structure (e.g. $[6,14,28,34])$. While most studies compare argumentation structure as described in RST [22], Stede et al. [34] examine the relationship between argumentation structure and discourse 
structure as conceived of in SDRT [2], which will be focal in our study. Annotations of their corpus of argumentative microtexts [27] indicate that edges of units realizing argumentative moves, which may consist of several elementary DUs, coincide with edges of clause-based elementary DUs realizing SDRTbased DRs in 63\% of all cases ([34], p. 1056). Depending on their frequency of occurrence in these data, the DRs Contrast, Elaboration, Continuation, Result and Explanation seem most relevant for argumentation, showing systematic variation in their correspondences with argumentative moves (cf. [34], p. 1057). But why should argumentative moves coincide with DRs only in some cases, but not in others, and what may be the cause for varying correspondences?

Peldszus and Stede argue that "distinguishing rhetorical structure [discourse structure in our terms] and argumentation structure is important for capturing the different aspects of a text's coherence on the one hand, and its pragmatic function on the other" ([28], p. 104). However, while argumentation structure and discourse structure approach argumentative discourse from distinct theoretical perspectives, their units of analysis may coincide. In instances of coinciding units of analysis, i.e. of coinciding stretches of discourse structure and argumentation structure, units are not only assigned discourse-anchored DRs by interlocutors at a local level, but have additional argumentative functions. In (1) analysed above, for instance, the argumentative genre of editorial guides interlocutors to assign the argumentative function of Rebuttal to DUs \#1/2 and \#1/3 related by Contrast; the DC but thus does not only signal Contrast, but fulfils the function of a linguistic indicator for the Attack move realized in \#1/3.

Many defining conditions of DRs are also employed in the definitions of argumentative moves. For instance, the defining condition of topic specification makes Elaboration particularly apt to realize Support moves, with the second DU adding further supportive detail to an argument or a supporting premise expressed in the first. However, Elaboration can also supply additional details that may result in the Rebuttal of an argument or of a supporting premise.

We may conclude that argumentation structure and discourse structure may coincide, and that overt signals may provide important cues for the interpretation of both. Against this background, the accommodation of genre-specific degrees of overtness with regard to the linguistic realization of DRs may be beneficial to annotation.

\section{Data and methodology}

To determine the impact of genre on the implicit vs. overt realizations of DRs, this paper compares DR realizations across two genres and two production modes. In a first step, we compare and contrast the realizations of DRs across two datasets of single-authored texts, from two genres, that are representative of free realizations of argumentative and narrative discourse - editorial and personal narrative to examine whether significantly different patterns of degrees of overtness are found between these genres; in a second step, the variation of degrees of overtness derived from this comparison is compared and contrasted to the variation of degrees of overtness in two datasets of co-constructed editorials and narratives discourse elicited in editing-based tasks. These co-constructed data are more constrained than single-authored data in that they are all based on the same original text, but they allow to evaluate under comparable conditions whether genre may account for systematic variation and produce genrepreferential degrees of overtness. The following sections provide further detail on data, annotation and methods of analysis. 


\subsection{Data}

Informed by previous studies that suggest differences in the linguistic realization of DRs between argumentative and narrative discourse (e.g. [11]), our contrastive approach compares data from the argumentative genre of editorial with data from the narrative genre of personal narrative. ${ }^{4}$

Narrative discourse and its realization within the genre of personal narrative are constrained by storyinternal configurations, such as number of characters, setting and plot, and by story-external, cultural configurations, such as spoken or written modes or participation format [10]. Following Labov [18], a personal narrative typically contains a reference to a single past event, introduced by a verbal phrase realized in a past tense, and a raison d'être. The past event hinges on a reportable and tellable personal experience, consisting of a series of events in the past that are contingent on one another and recounted from a 1st- or 3rd-person perspective. The sequence of events in the series, generally reflecting the temporal sequence of events, is open to some variation with regard to length and tense shift, but needs to be in accordance with story-internal and -external constraints.

Similar to argumentative discourse, narrative discourse appears to have preferences with respect to overt realization of the connectedness between its constitutive units: chronological sequence between events is typically signalled with clause-initial adverbials of time [7], while DCs such as and, then and next may serve as "segmentation markers" signalling topic continuity and discontinuity [5], indicate shifts in the deictic centre [31] and express involvement on the side of the writer [10]. Editorials and personal narratives are thus expected to exhibit differences with respect to their overt vs. implicit realizations of DRs.

Table 2 summarizes the structure of our dataset. For single-authored data, we draw on English datasets employed by [32,33], comprising a set of 9 editorials from British newspaper The Guardian, and a set of 10 short personal narratives from British university students. Co-constructed data, also collected for previous studies [11,21] contain 9 argumentative and 9 narrative texts jointly produced by 18 participant dyads ( 9 per genre) of native speakers of English. They were obtained through an editing-based task (cf. Section 4.2) designed to elicit, in a controlled fashion, comparable data that permits evaluation, specification and testing of genre-preferential variation in the linguistic realization DRs derived from the analysis of single-authored data. ${ }^{5}$

Table 2

Narrative and argumentative datasets

\begin{tabular}{lccr}
\hline Data & Editorials & Personal narratives & Total $N$ \\
\hline Single-authored & 9 texts & 10 texts & \\
$N$ of words & 4,826 & 4,551 & 9,377 \\
Co-constructed & 9 texts & 9 texts & \\
$N$ of words & 1,838 & 1,864 & 3,702 \\
Total $N$ & 6,664 & 6,415 & 13,079 \\
\hline
\end{tabular}

\footnotetext{
${ }^{4}$ Using narrative discourse as a reference genre is advantageous in light of its conceptual closeness to expository discourse, which according to previous research differs from argumentative discourse with respect to the production and interpretation of discourse coherence in general and DRs in particular (e.g. [3,4,29]).

${ }^{5}$ The single-authored narratives were collected by N.M. Fronhofer for her PhD project on a contrastive analysis of the linguistic realization of emotions. The editing-based tasks were carried out by students and staff of the University of Augsburg and the University of Glasgow.
} 


\subsection{Editing-based tasks}

To elicit argumentative and narrative data comparable to single-authored data, the editing-based tasks employed two 'bare' texts adapted from an editorial and a personal narrative written by single authors. These texts were stripped of all extra-clausal constituents such as parentheticals, adverbials of time or place and DCs [21], but retained original sequence and default configuration of events. Strings of bare DUs of this kind presumably reflect a minimum of propositional content and allow for maximal diversity with respect to the interpretation of DR potentials that they refer to indexically.

The editing-based task asked participants to collaboratively add linguistic material to the bare text or alter it in order to create a well-formed text of the specified genre (bare texts and instructions are reproduced in the Appendix). Relying exclusively (1) on sequential ordering of DUs and (2) on semantics of intra-clausal material encoding potential DRs, they had to negotiate possible interpretations of the relations between the DUs to agree on a specific DR, and alter the text where necessary. This allows conclusions about (a) where coherence strands encoded in the bare DUs were perceived to carry defining conditions that were considered sufficient to specify a DR, (b) where they were considered to be less than sufficient and therefore required additional encoding and signalling to specify a DR, and (c) where they were considered to be underspecified for a DR, but still sufficient, thus allowing multiple assignment.

\subsection{Annotation}

Annotations of both single-authored and co-constructed texts were carried out in line with the theoretical considerations outlined in Section 2. Two annotators independently segmented the text into non-overlapping clause-based elementary DUs and, in line with the taxonomy illustrated in Table 1 (cf. Section 2.1), coded them for coordinating DRs (Continuation, Narration, Contrast and Background) and subordinating DRs (Elaboration, Explanation and Comment). ${ }^{6}$ Annotations also considered the adjacent vs. non-adjacent positioning of DUs realizing DRs, and DRs' implicit vs. overt linguistic realizations as reflected in their encoding in coherence strands, and signalling with DCs, metacomments and noncongruently configurated theme zones. Deviating codings were negotiated and aligned at all relevant stages of analysis.

\subsection{Analysis}

To determine the impact of genre on the implicit vs. overt realizations of DRs, a series of quantitative analyses examine

(1) whether the overt vs. implicit realization of DRs as reflected in degrees of overtness per DR and genre in single-authored argumentative and narrative texts reveals significant differences between the genres that may corroborate the existence of genre-preferential DR realizations. To test whether genre has any significant effect upon overt vs. implicit DR realization, an independent one-way Analysis of Variance ${ }^{7}$ compares variability within and between mean degrees of overtness per DR in texts of both genres. In view of unequal sample sizes and variances between genres (a consequence of having to use different texts), differences in average degrees of overtness between genres are compared with a weighted independent measures (Welch's) $t$-test.

\footnotetext{
${ }^{6}$ Assuming a different sequential organization, but conceptually representing the same relation, Result has been conflated with Explanation to facilitate annotation.

${ }^{7}$ Repeated measures ANOVA is not possible since degree of overtness does not fulfil the requirements of a repeated measures factor.
} 
(2) whether there are significant differences between single-authored and co-constructed texts within the two genres with respect to degrees of overtness. This may corroborate or refute the assumed impact of genre on DR realization depending on whether co-constructed data show similarities or deviations from the genre-preferential variation derived from analysis of single-authored data. As above, an independent one-way ANOVA examines the general influence of genre on degrees of overtness in co-constructed data; to compare the degrees of overtness of individual DRs in singleauthored data with those in co-constructed data, one-sided $t$-tests compare dyads' means with the expected means derived from single-authored data.

(3) in what way degrees of overtness behave with respect to adjacent vs. non-adjacent positioning across genres and modes of production. Chi-squared tests accounting for the low number of categories examine differences between genres and modes of production.

Because some DRs (Background and Narration) do not occur in all genres and/or modes of production, they may not be considered in all quantitative analyses - especially in statistical analyses that can only take into account DRs that occur in both modes of production/genres - but will be considered in reports on overall degrees of overtness.

\section{Results}

Annotation of single-authored and co-constructed narrative and argumentative texts finds 1,485 DRs across genres and production modes, with DRs in single-authored texts making up $80 \%$ of all DRs $(N=1,189)$. Despite unequal sample sizes, proportional representation of counts reveals systematic differences both between the genres under consideration and between the two modes of production, as reflected in Fig. 1: within and across production modes, narratives appear to show an overall higher degree of overtness than editorials. Moreover, the overall degrees of overtness seem to be lower in coconstructed texts than in single-authored texts.

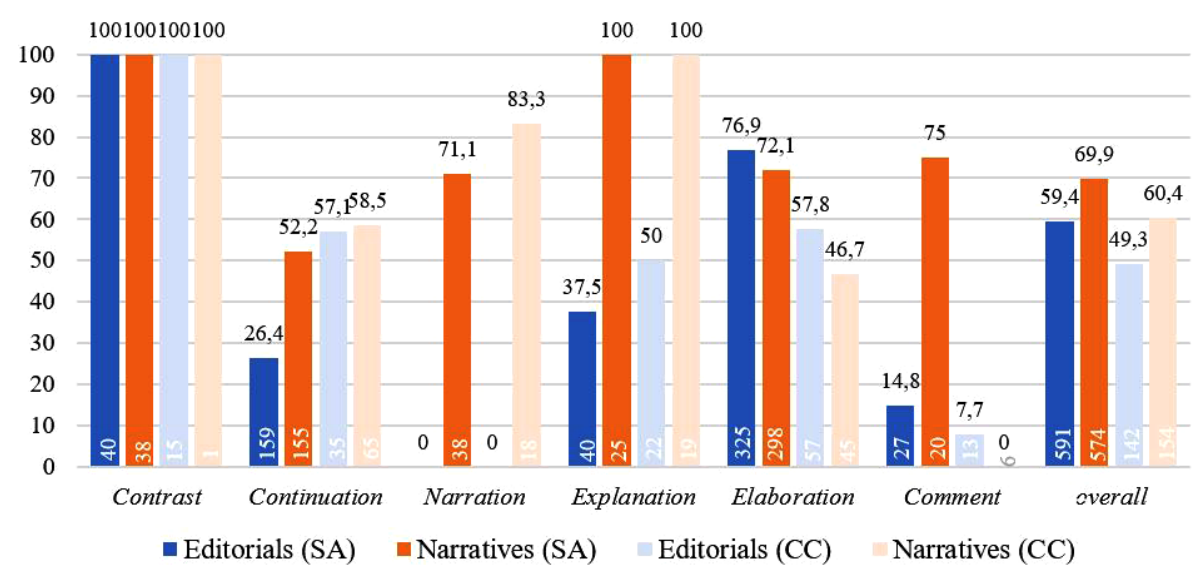

Fig. 1. Degrees of overtness in single-authored and co-constructed editorials and narratives. ${ }^{8}$

\footnotetext{
${ }^{8} \mathrm{Ns}$ on the vertical axis and tops of columns indicate degrees of overtness as percentages. Ns provided in the columns represent raw Ns of occurrence. Background, which is not individually considered in the analysis, is not represented.
} 
Table 3

Differences per DR in means of degrees of overtness between single-authored editorials and single-authored narratives

\begin{tabular}{lccccc}
\hline DR & $\begin{array}{c}\text { Means } \\
\text { (Editorials) }\end{array}$ & $\begin{array}{c}\text { Means } \\
\text { (Narratives) }\end{array}$ & $t$ & $d f$ & $p$ \\
\hline Contrast & 1 & 1 & - & - & - \\
Continuation & 0.24 & 0.54 & 4.65 & 16.96 & $<0.001$ \\
Explanation & 0.45 & 0.96 & 2.84 & 7.50 & $<0.05$ \\
Elaboration & 0.78 & 0.75 & -2.60 & 16.69 & $<0.05$ \\
Comment & 0.12 & 0.84 & 5.88 & 14.99 & $<0.0001$ \\
\hline
\end{tabular}

The test value $t$ is an indicator of significant difference between the means of two populations (here: genres). Narration, due to zero occurrences in the argumentative data, is not considered here.

\subsection{Degrees of overtness in single-authored data}

An independent one-way ANOVA comparing degrees of overtness in single-authored editorials and narratives finds a significant impact of genre $(F(1,17)=51.82, p<0.0001)$, with narratives exhibiting higher overall degrees of overtness (79.8\%) than editorials (51.6\%). This is also supported by a weighted independent measures $t$-test between genres that is not confined in terms to DRs and considers all realizations $(t(218.5)=3.20, p<0.01)$. Proportionally, the distribution of overt realizations is found to be significantly unequal in both genres, i.e. at least one DR shows a different distribution than the others $\left(\chi^{2}(5)=51.838, p<0.0001\right.$ for personal narratives; $\chi^{2}(4)=170.65, p<0.0001$ for editorials). Independent measures $t$-tests between genres for individual DRs are reported in Table 3. These disclose significant differences between the genres for Comment, Continuation, Explanation and Elaboration. All show lower degrees of overtness in editorials than in narratives, with the exception of Elaboration, which is realized more overtly in editorials. Contrast is realized overtly throughout in both genres.

\subsection{Degrees of overtness in single-authored and co-constructed data}

An independent one-way ANOVA comparing co-constructed editorials and narratives finds that differences between genres in terms of degrees of overtness are also significant in the co-constructed data $(F(1,17)=8.13, p<0.05)$, with the narrative genre showing a higher degree of overtness $(64.9 \%)$ than the argumentative one $(61.4 \%)$. This is again confirmed by a weighted independent measures $t$-test with $t(196.36)=-2.69, p<0.01$. Proportion tests again provide evidence that the distribution of overt realizations of at least one DR differs from the overall distributions of all others' in both editorials $\left(\chi^{2}(4)=27.122, p<0.0001\right)$ and narratives $\left(\chi^{2}(5)=25.331, p<0.001\right)$.

Table 4 reports the results of $t$-tests between expected means derived from single-authored data and the means per dyad in co-constructed data; these provide information on whether the degrees of overtness that are found for individual DRs in co-constructed texts correspond to those found in single-authored texts. As in single-authored texts, Contrast is realized overtly in co-constructed datasets throughout. In data for the argumentative genre, mean degrees of overtness for DRs Explanation and Comment in co-constructed texts are comparable to those from single-authored data. For DRs Continuation and Elaboration, however, mean degrees of overtness differ significantly from those expected on the basis of single-authored texts. In the narrative genre, mean degrees of overtness for DRs Continuation, Narration and Explanation in co-constructed texts are comparable to those from single-authored data, but show significant differences with DRs Elaboration and Comment. 
Table 4

Differences per DR between means of degrees of overtness of co-constructed texts and expected means from single-authored texts

\begin{tabular}{|c|c|c|c|c|c|c|}
\hline$\overline{\mathrm{DR}}$ & Genre & $\begin{array}{c}\text { Means } \\
\text { (co-constr.) }\end{array}$ & $\begin{array}{c}\text { Means } \\
\text { (expected) }\end{array}$ & $t$ & $d f$ & $p$ \\
\hline \multirow[t]{2}{*}{ Contrast } & Editorials & 1 & 1 & - & - & - \\
\hline & Narratives & 1 & 1 & - & - & - \\
\hline \multirow[t]{2}{*}{ Continuation } & Editorials & 0.57 & 0.24 & 3.05 & 8 & $<0.05$ \\
\hline & Narratives & 0.59 & 0.54 & 0.62 & 9 & 0.55 \\
\hline \multirow[t]{2}{*}{ Narration } & Editorials & $\mathrm{n} / \mathrm{a}$ & $\mathrm{n} / \mathrm{a}$ & $\mathrm{n} / \mathrm{a}$ & $\mathrm{n} / \mathrm{a}$ & $\mathrm{n} / \mathrm{a}$ \\
\hline & Narratives & 0.83 & 0.70 & 1.20 & 9 & 0.26 \\
\hline \multirow[t]{2}{*}{ Explanation } & Editorials & 0.37 & 0.45 & -0.59 & 8 & 0.57 \\
\hline & Narratives & 0.95 & 0.96 & -0.19 & 9 & 0.86 \\
\hline \multirow[t]{2}{*}{ Elaboration } & Editorials & 0.39 & 0.78 & -10.04 & 8 & $<0.0001$ \\
\hline & Narratives & 0.39 & 0.75 & -4.39 & 9 & $<0.005$ \\
\hline \multirow[t]{2}{*}{ Comment } & Editorials & 0.06 & 0.12 & -1.07 & 8 & 0.32 \\
\hline & Narratives & 0.14 & 0.84 & -4.91 & 6 & $<0.005$ \\
\hline
\end{tabular}

Table 5

Degrees of overtness in adjacently and non-adjacently realized DRs in single-authored and co-constructed editorials and narratives

\begin{tabular}{|c|c|c|c|c|c|c|c|c|}
\hline \multirow[t]{2}{*}{$\overline{\mathrm{DR}}$} & \multicolumn{2}{|c|}{$\begin{array}{c}\text { Editorials } \\
\text { (single-authored) }\end{array}$} & \multicolumn{2}{|c|}{$\begin{array}{c}\text { Narratives } \\
\text { (single-authored) }\end{array}$} & \multicolumn{2}{|c|}{$\begin{array}{c}\text { Editorials } \\
\text { (co-constructed) }\end{array}$} & \multicolumn{2}{|c|}{$\begin{array}{c}\text { Narratives } \\
\text { (co-constructed) }\end{array}$} \\
\hline & $\%$ & $\overline{(\text { total } N)}$ & $\%$ & $\overline{(\operatorname{total} N)}$ & $\%$ & $($ total $N)$ & $\%$ & $\overline{(\text { total } N)}$ \\
\hline adjacent & 67.8 & (468) & 73.2 & $(462)$ & 51.2 & (121) & 66.1 & $(127)$ \\
\hline non-adjacent & 27.6 & (123) & 55.2 & (136) & 38.1 & (21) & 48.1 & (27) \\
\hline overall & 59.4 & (591) & 69.9 & (598) & 49.3 & (142) & 60.4 & (154) \\
\hline
\end{tabular}

For single-authored narratives, Table 5 also factors in the DR of Background, which is not individually considered in the analysis and therefore not represented in Fig. 1, and yields, for this reason, a higher overall $N$ of DRs than Fig. 1.

\subsection{Degrees of overtness and adjacency}

Table 5 indicates how degrees of overtness behave across genres and modes of production with respect to adjacent vs. non-adjacent positioning of DUs realizing DRs. Degrees of overtness vary considerably between adjacently and non-adjacently positioned $\mathrm{DRs}^{9}$ in single-authored data of both genres $\left(\chi^{2}(1)=\right.$ 64.92, $p<0.0001$ for editorials; $\chi^{2}(1)=15.96, p<0.0001$ for narratives), with less variation in the co-constructed ones $\left(\chi^{2}(1)=1.24, p=0.27\right.$ for editorials; $\chi^{2}(1)=2.05, p=0.15$ for narratives).

While the overall proportion of adjacently and non-adjacently positioned DRs is relatively homogeneous across all data (80.3\% in argumentative vs. $78.2 \%$ in narrative data), genres differ with respect to their preferences for degrees of overtness of DRs in (non-)adjacently positioned DUs. In both adjacently and non-adjacently positioned DRs in narrative data, higher degrees of overtness are encountered than in the argumentative data. In single-authored editorials, overt realization is preferred in adjacently positioned DRs, but dispreferred in non-adjacently positioned DRs. Single-authored narratives always favour overt realizations, with degrees of overtness being higher in adjacently than in non-adjacently positioned DRs. Co-constructed data repeat the overall tendencies of single-authored data, but have lower

\footnotetext{
${ }^{9}$ For simplification, the remainder of the paper employs the technically incorrect formulation "(non-)adjacently positioned DR" to refer to (non-)adjacently positioned DUs realizing DRs.
} 
overall degrees of overtness, except for non-adjacent DRs in editorials, which show a higher degree of overtness in co-constructed than in single-authored data.

While degrees of overtness of adjacent DRs show no remarkable variation based on genre, neither for single-authored nor for co-constructed data, significant genre differences in degrees of overtness are encountered for non-adjacent DRs within single-authored data, with narratives showing significantly stronger preferences than editorials for overt realizations of DRs in non-adjacent position $\left(\chi^{2}(1)=8.92\right.$, $p<0.005)$. These differences are not discovered with co-constructed texts $\left(\chi^{2}(1)=1.17, p=0.28\right)$.

\subsection{Summary}

The results of quantitative analyses of single-authored data reveal systematic differences in the variation of degrees of overtness between editorials and personal narratives: in general, argumentative data yield lower degrees of overtness than narrative data, with differences most noticeable for Comment, Continuation and Explanation, and within adjacently positioned DRs; Elaboration is the only DR with a significantly higher degree of overtness in single-authored editorials.

Comparisons of single-authored texts with co-constructed texts show that joint productions are less overt than the single-authored data, but mirror their overall preferences for the encoding and signalling of DRs: with the exception of Elaboration, all DRs are realized in argumentative data with lower degrees of overtness than in narrative data; Contrast is realized overtly throughout, irrespective of genre. Tests of individual DRs per genre reveal that mean degrees of overtness in co-constructed narratives echo those of single-authored narratives for Contrast, Continuation, Narration and Explanation, but are significantly lower for Elaboration and Comment. Within argumentative data, co-constructed editorials mirror single-authored editorials with respect to the realization of Contrast, Explanation and Comment, but Elaboration is realized significantly less overtly, and Continuation significantly more overtly, than in single-authored texts of a comparable genre.

\section{Discussion}

Results from the quantitative analyses show that the systematic differences in the variation of degrees of overtness between single-authored editorials and personal narratives largely also bear out in the coconstructed data: while joint productions are less overt, they tend to display similar preferences for the encoding and signalling of DRs. We assume that the lower degrees of overtness in the co-constructed data may result from the particular nature of the editing based tasks, requiring participant dyads to negotiate their linguistic realization of the connectedness of the bare DUs. The "final product" of their jointly edited texts does not reflect instances where the bare units were considered to be sufficiently connected, but only those where they required additional signals. This seems to hold for all DRs but Contrast, which is overt throughout the data, and Continuation, which prefers implicit realization in the single-authored, but not in the co-constructed editorials. Typical realizations of Continuation in the argumentative data are illustrated in excerpts (4) and (5), respectively representing single-authored and co-constructed realizations. In (4), Continuation between DUs 1 and 2 is realized implicitly, with referential continuity encoded in pronoun 'they' and temporal continuity including continuity in modality ('can help' - 'already consider'). In (5), Continuation, which was already encoded in temporal-aspectual continuity as well as in several strands of lexical coherence, was supplemented by the participant dyad with temporal adverbials 'for decades' and 'over the last few years', thus creating parallel non-congruently configurated theme zones which additionally signal the DR overtly. 
Table 6

Genre-preferential degrees of overtness

\begin{tabular}{lcc}
\hline DR & Editorials & Narratives \\
\hline Contrast & 100 & 100 \\
Continuation & 32 & 54 \\
Narration & $\mathrm{n} / \mathrm{a}$ & 75.9 \\
Explanation & 41.9 & 100 \\
Elaboration & 74 & 68.8 \\
Comment & 12.5 & 57.7 \\
adjacent DR & 64.3 & 71 \\
non-adjacent DR & 29.2 & 53.7 \\
overall & 57.4 & 67.9 \\
\hline
\end{tabular}

(4) Obesity/1 [This is where local councils can help.]

Obesity/2 [They already consider APPLICATIONS for alcohol licenses carefully,]


CLINE]

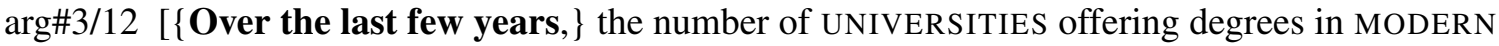
LANGUAGE has PLUMMETED.]

It remains unclear why the preferences with respect to the realization of Continuation in editing-based tasks differ from preferences of single-authored texts in the dataset of editorial to the extent that has been observed. Participant dyads may be attempting to be more precise in their argumentation, whereas authors of single-authored media texts might wish to remain diplomatically unclear at some stages in their argument.

Ignoring Continuation as an outlier that is due to the format of production rather than to the genre, the variation in degrees of overtness that is derived from the analysis of the single-authored editorials arguably also bears out in co-constructed texts. This corroborates the hypothesis that genre functions as an important constraint on DR realization leading to genre-specific degrees of overtness. The variation of degrees of overtness across modes of production may thus be considered as reflective of genre-preferential patterns of DR realization for the genres examined. These genre-preferential degrees of overtness are summarized in Table 6, based on degrees of overtness calculated across modes per genre. The following sections discuss the genre-preferential degrees of overtness of individual DRs with a focus on the DRs' roles in argumentative discourse.

\subsection{Contrast}

Whereas all other DRs show variable patterns of realization, Contrast in our data is always realized overtly with non-propositional material, i.e. DCs, metacomments and/or pragmatic word order. Since it is additionally encoded in coherence strands throughout, most prominently in antonymic lexical relations and shifts in temporal continuity, it is overspecified by default. This becomes particularly clear if we take into consideration that in both narrative and argumentative editing-based tasks Contrast is always supplemented with overt signals despite generally being encoded in proposition-anchored coherence strands of the bare DUs.

A typical example is provided in (6), an argumentative sequence from a co-constructed editorial in which the Claim in $\arg \# 2 / 2$ is rebutted through arg\#2/3. In carrying out their task, the dyad who produced 
excerpt (6) supplements Contrast, which is encoded in topic and referential continuity ('London' - 'it'), temporal discontinuity ('was' - 'is') and lexical antonymy ('dowdy'; 'stale' - 'exciting'), with two overt signals: contrastive DC 'but' and two parallel fronted temporal adjuncts and lexical antonyms 'in the past' and 'now', which additionally encode temporal discontinuity.

(6) $\arg \# 2 / 2$ [ [In the past $\}$ London was a DOWDY place of tea-houses and STALE rock cakes.] $\arg \# 2 / 3$ [\{But now, $\}$ it's MUCH MORE EXCITING.]

Overspecified Contrast may be preferred by argumentative genres for reasons of salience since Contrast, in view of its defining condition of semantic dissimilarity, may take over important argumentative Attack functions like antithesis, realizing Undercut moves, or concession, realizing Rebuttal as in (6). However, the consistent supplementation of coherence strands with overt signals in both editorials and narratives suggests that realization of Contrast is overspecified by default; in other words, its realization is not primarily constrained by the genre, but by its semantics, leading to an overspecified, and thus overt, realization regardless of context.

\subsection{Elaboration}

Apart from Contrast, Elaboration is the only DR that shows relatively similar degrees of overtness across genres, exhibiting preferences for overt realization in both editorials and narratives, with a slightly higher degree of overtness in editorials. In Elaboration, a DU offers additional information about one of the referents in a preceding DU, making it a fairly prominent DR in argumentative discourse. There, it may be assigned the argumentative functions of adding supportive detail or providing an example, thus correlating with Support moves, or of adding additional detail that may result in the rebuttal of an argument, thus correlating with Rebuttal moves.

In excerpt (7), for instance, Elaboration between 1 and 2 is encoded in mereological topic specification between 'Hizbullah' and 'the Bekaa raid', temporal/aspectual overlap ('is' - 'may have been') and various strands of lexical coherence, and signalled overtly with the fronted adverbial 'as such', particularizing the role of the Bekaa raid. Within the argumentative sequence, the constraints of the genre guide interlocutors to assign the argumentative function of Support to the pair of DUs related through Elaboration.

(7) Lebanon/1 [[There is no doubt that HizBULLAH sees itself as the victor in the CONFLICT] [and this in itself is a provocation to an ISRAELI government which is under pressure at home to show it can still protect its territory.]]

Lebanon/2 [As such, the BEKAA raid may have been a symbolic exception to a policy of compliance with the UN RESOLUTION, rather than a disturbing indication of flagrant BREACHES to come.]

Elaboration having similar degrees of overtness across genres may not necessarily be due to its semantics, as with Contrast, but rather to both genres requiring overt signalling of the DR in order to ensure activation of the relevant defining conditions and thus assignment of a DR that introduces a lower level of discourse. In (7), for example, the non-signalling of Elaboration might guide interlocutors to assign the DR Continuation with the argumentative function of "multiple support" [26], that is of DU 1 and 2 realizing two independent arguments on the same level rather than a sequence of Claim and Support move.

Similarly, Elaboration may need to be marked overtly in narrative discourse to ensure the speakerintended interpretation. This is shown in (8), where adverbial 'more particularly' signals the defining 
condition of topic specification for Elaboration overtly, with narr\#5/4 introducing a lower level of discourse rather than keeping the discourse on the same level - a potential interpretation of connectedness of the bare DUs that is reflected in excerpt (9), where the dyad assigned Continuation.

(8) narr\#5/3 [His job involves working for SENSHU as an ENGLISH LANGUAGE COORDINATOR.] narr\#5/4 [\{More specifically, $\}$ he provides TRANSLATIONS and revisions to CORRESPONDENCES sent between SENSHU and its affiliated universities, along with teaching weekly tutorial ENGLISH courses.]

(9) nar\#9/3 [ $\underline{\mathrm{He}}$ works for SENSHU as an ENGLISH LANGUAGE COORDINATOR]

nar\#9/4a [ $\{$ and (he) also $\}$ provides TRANSLATIONS and revisions to CORRESPONDENCES sent between $\underline{\text { SENSHU }}$ and other universities,]

\subsection{Continuation}

Unlike Elaboration, Continuation, which is defined by topic identity in the DUs it relates, shows differences between the genres under consideration. Whereas the genre of editorial prefers Continuation to be realized implicitly with relatively low degrees of overtness, narratives show a preference for higher degrees of overtness (cf. excerpt (9)). Although Continuation, unlike Contrast, Elaboration or Explanation/Result, does not seem to show any clear co-occurrences with argumentative moves according to Stede et al. [34], its frequent occurrences in our argumentative data suggest that it is nevertheless prominent in argumentation.

The typically implicit realization of Continuation in the argumentative genre is illustrated in excerpt (4), above, and in (10), from a co-constructed text. Although one motivation for Continuation to be realized implicitly in editorials may be the author's preference for remaining diplomatically unclear (see above), data suggests that the argumentative genre may generally prefer the defining conditions of Continuation to be encoded in coherence strands and lexical material only. An example is shown in (10), where 'an additional concern' indexes Continuation, supplementing temporal/aspectual coherence ('has plummeted' - 'has hit') and lexical coherence strands. On the level of argumentation, the lexical index 'an additional concern' fulfils the function of a linguistic indicator for a multiple support structure in which arg\#3/12 and arg\#3/14 realize independent arguments for the conclusion that British students are losing interest in foreign language learning.

(10) $\arg \# 3 / 12$ [\{Over the last few years, $\}$ the number of UNIVERSITIES offering degrees in MODERN LANGUAGE has plummeted.]

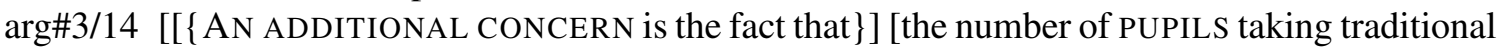
MODERN FOREIGN LANGUAGES for their A-Levels has hit its lowest point since the mid-90s.]]

\subsection{Explanation/Result and Comment}

The preferences of argumentative and narrative genres seem to differ most for Explanation (conflated in our analyses with Result) and Comment. Defined by causal connectivity and temporal order, Explanation and Result express a cause-result relationship between two DUs that may argumentatively function as a sequence of Claim and Support move (Explanation) or of Support move and Claim (Result). Our data suggests a preference of Explanation for implicit realization in the genre of editorial, but for exclusively overt realization in narratives, as illustrated in excerpts (11) and (12), respectively from an argumentative and a narrative context. With referential continuity ('It' - 'Mahmoud Ahmadinejad') and 
temporal and aspectual coherence ('is operating' - 'has been conducting') as the only carriers of defining conditions of Explanation linking DUs 1 and 2, (11) shows an argumentative sequence of Claim and Support and reflects the preferred implicit realization of the DR in the editorials. Explanation in (12), encoded in referential, temporal and aspectual continuity, and is signalled through discourse connective because, reflecting its preferred realization in the narrative data.

(11) Iran/1 [[It (Iraq) is certainly operating -] [as Chatham House puts it in a timely new report,] [- in an atmosphere of "confident ease."]]

Iran/2 [[Mahmoud Ahmadinejad, the hardline president, has been conducting an energetic charm offensive] [in which much attention has been paid to his twinkling eyes and domestic popularity]]

(12) 1 [Well would you believe it; ]

2 [I have failed the EXAM again.]

3 [[I wouldn't have minded] [because it's not such a favourite SUBJECT of mine,] [but ... ]]

Genre-preferential degrees of overtness that are very similar to those of Explanation were encountered for Comment, which is defined by a DU selecting another one as its topic. In argumentative genres, Comment may be assigned the argumentative function of supporting a premise or argument or of concluding. Like Explanation, it strongly favours implicit realization in the editorials, and has a preference for overt realization in the narratives. We assume that the different preferences of narrative and argumentative genre with respect to the overt vs. implicit realization of Explanation and Comment arise from genrespecific expectations and their respective inferential schemata [19].

In narrative discourse, interlocutors by default consider a sequence of DUs as representing temporally unfolding events that are linked through the DR Narration; DRs that indicate deviations from this narrative sequence, such as Comment and Explanation, thus require overt marking, and lead throughout our data to significantly higher degrees of overtness for these DRs in narrative texts in comparison to argumentative texts. In argumentative discourse, on the other hand, interlocutors are prone to producing/interpreting sequences of DUs as being linked causally (rather than temporally or continuatively, for instance). For this reason, it seems redundant to signal DRs such as Explanation or Comment fulfilling argumentative functions of Support overtly in argumentative discourse.

\subsection{Adjacency}

The impact of genre-specific constraints and expectations for argumentative and narrative discourse with respect to the linguistic realization of DRs and their processing is also reflected in the preference for significantly higher degrees of overtness in the realization of non-adjacently positioned DRs in narrative data as compared to argumentative data: we assume that for personal narratives, non-adjacently positioned DUs realizing DRs prefer overt realization because deviations from the default chronological story line need to be accounted for. The fact that editorials appear to prefer significantly lower degrees of overtness in the realization of non-adjacent DRs, on the other hand, may indicate that the constraints of argumentative discourse guide interlocutors to expect arguments and their constitutive units to not be linearized in a straightforward manner; if lower levels of argumentation are introduced within the argumentative sequence, for instance by Elaboration and Explanation/Result, the overt signalling of these DRs thus may be considered redundant. 


\subsection{Summary}

Overall, the systematic variation in the overt vs. implicit realization of DRs appears to be influenced mainly by (1) the semantics of DRs, leading to default realizations irrespective of genre, as is the case with Contrast, and (2) genre, leading to genre-preferential degrees of overtness, as is the case with Explanation/Result, Comment, Continuation and Elaboration. Genre-preferential degrees of overtness in the data seem to result from the production and interpretation of DRs being constrained by genres and their inferential schemata, which specify the typical ways for the encoding and signalling of DRs in context that interlocutors may act in accordance or disaccordance with, as is reflected in genre-internal and participant-specific variation.

For the argumentative genre of editorial, the following constraints for the production and interpretation of DRs seem to hold: because the constraints of argumentation guide interlocutors to produce and interpret sequences of DUs as being linked causally, Continuation, Explanation/Result and Comment do not need to be signalled overtly unless a particular argumentative goal is intended at a particular stage in the discourse which requires them to be signalled with discourse connectives and/or pragmatic word order. Elaboration, however, requires overt signalling as it introduces a lower level in the argumentation, e.g. to function as Support that further develops an argument, and needs to be distinguished from Continuation, which would keep the argumentation on the same level, e.g. by introducing an independent argument in multiple Support. Contrast is overspecified by default.

\section{Conclusion}

Conceiving of DRs as sociocognitive constructs that are encoded in coherence strands and may additionally be signalled overtly with discourse connectives, metacomments and/or pragmatic word order, this study set out to establish whether argumentative discourse shows genre-specific preferences for overt vs. implicit realizations of DRs by means of a doubly contrastive approach that compared the argumentative genre of editorial with the narrative genre of personal narrative within and across two production modes. Analyses show systematic differences in the variation of degrees of overtness between editorials and personal narratives, supporting the hypothesis of genre-preferential linguistic realization of DRs for all DRs except for Contrast. The primary constraint for linguistic realization of Contrast is semantics, which leads to this DR being encoded and additionally signalled by default. Genre may thus be assigned the status of a macro frame that constrains the production and interpretation of DRs with respect to varying degrees of overtness. In the argumentative genre of editorial, interlocutors tend to produce and interpret sequences of DUs as being linked causally, which is why the DRs of Explanation, Continuation and Comment are signalled overtly only if the local argumentative need arises. Elaboration, by contrast, is signalled with higher degrees of overtness as it introduces a lower level in the argumentative sequence and is thus relevant to argumentative coherence.

As regards annotation, the genre-specific realizations of DRs may contribute to the development of algorithms for the automatic assignment of DRs that may factor in genre-preferential degrees of overtness of DRs and their most frequent overt signals and coherence strands, allowing discourse structure annotation tools to accommodate a higher degree of context- and genre-sensitivity. What is more, due to structural coincidences between argumentative structure and discourse structure, the study of genrespecific variation of overt vs. implicit DR realization may also be relevant to approaches in Argument Mining that aim to predict argumentation structure from discourse structure. Units of analysis of discourse structure and argumentation, we argue, may overlap if the constraints of argumentative discourse 
guide the assignment of argumentative functions to DUs related by a DR. If annotation is trained specifically on text portions in which the two structures overlap, the preferred coherence strands and overt signals of DRs in the training data may become operationalizable as cues for the automatic annotation of argumentation.

\section{Appendix A. Editing-based task - argumentative discourse}

The following 15 clauses form the backbone of a commentary from the Guardian. Please transform them into a well-formed coherent whole. You may add or delete any linguistic material which you consider necessary, but you may not change the order of the given units:

\section{The solitary monoglots}

1. the British seem set on isolation from the world

2. London was a dowdy place of tea-houses and stale rock cakes

3. it's much more exciting

4. I can hear people speaking in all the languages of the world

5. was that Pashto or Hindi

6. I can differentiate Polish from Lithuanian

7. I delight in hearing them mingled with snatches of French, German, Spanish, Italian, Japanese

8. London has become the capital of linguistic diversity

9. one important group seems to be leaving itself out

10. students

11. foreign language learning at Britain's schools has been in decline

12. the number of universities offering degrees in modern languages has plummeted

13. an inquiry is under way

14. the number of teenagers taking traditional modern foreign languages at A-level fell to its lowest level since the mid-90s?

15. it's a paradox

\section{Appendix B. Editing-based task - narrative discourse}

The following 19 clauses form the backbone of a short narrative adapted from the University of $\mathrm{Ne}$ braska website. Please transform them into a well-formed coherent whole. You may add or delete any linguistic material which you consider necessary, but you may not change the order of the given units:

\section{Education abroad - student stories}

\section{John Smith, College of Business Administration (CBA)}

Senshu University, Tokyo, Japan, spring 2012

1. John Smith jetted off to Japan to start his career

2. He studied abroad at Senshu University

3. Smith works for Senshu as an English language coordinator

4. He provides translations and revisions to correspondences sent between Senshu and its affiliated universities, along with teaching weekly tutorial English courses 
5. Smith was exposed to Japanese culture through a family friend

6. He began to study the Japanese language

7. He has had a life-long interest in Japan

8. He prepared to work in the world's third largest economy

9. He felt his acquisition of the Japanese language would be in vain

10. He didn't have a strong grasp of basic business concepts and terminology

11. He decided on majoring in international business

12. He studied abroad through the College of Business Administration-sponsored exchange program with Senshu University

13. Smith learned how to move past cultural differences

14. He learned how to build very meaningful relationships

15. He became aware of cultural filters

16. Cultural filters affect someone's paradigm and viewpoint of certain situations

17. He learned more and more about the general viewpoints of Japanese people

18. He worked for the CBA Office of Undergraduate Programs as the Senshu study abroad student coordinator

19. The relationship he established with Senshu University is what led to him being offered the job prior to graduation

\section{References}

[1] J.C. Anscombre and O. Ducrot, L'Argumentation dans la Langue, Mardaga, Brussels, 1983.

[2] N. Asher and A. Lascarides, Logics of Conversation, Cambridge UP, Cambridge, 2003.

[3] I. Berzlánovich, M. Egg and G. Redeker, Coherence structure and lexical cohesion in expository and persuasive texts, in: Constraints in Discourse 3. Representing and Inferring Discourse Structure, A. Benz, M. Stede and P. Kühnlein, eds, Benjamins, Amsterdam, 2012, pp. 137-164. doi:10.1075/pbns.223.06ber.

[4] I. Berzlánovich and G. Redeker, Genre-dependent interaction of coherence and lexical cohesion in written discourse, Corpus Linguistics and Linguistic Theory 8(1) (2012), 183-208.

[5] Y. Bestgen and W. Vonk, The role of temporal segmentation markers in discourse processing, Discourse Processes 19(3) (1995), 385-406. doi:10.1080/01638539509544924.

[6] E. Cabrio, S. Tonelli and S. Villata, From discourse analysis to argumentation schemes and back: Relations and differences, in: Computational Logic in Multi-Agent Systems, 14th International Workshop, CLIMA XIV, Proceedings, Corunna, Spain, September 16-18, 2013, J. Leite, T.C. Son, P. Torroni, L. van der Torre and S. Woltran, eds, 2013, 1-17.

[7] J. Costermans and Y. Bestgen, The role of temporal markers in the segmentation of narrative discourse, CPC: European Bulletin of Cognitive Psychology 11 (1991), 349-370.

[8] J. Eckle-Kohler, R. Kluge and I. Gurevych, On the role of discourse markers for discriminating claims and premises in argumentative discourse, in: Proceedings of the 2015 Conference on Empirical Methods in Natural Language Processing, Lisbon, Portugal, 2015.

[9] A. Fetzer, Theme zones in English media discourse. Forms and functions, Journal of Pragmatics 40(9) (2008), 15431568. doi:10.1016/j.pragma.2008.04.016.

[10] A. Fetzer, Small stories in political discourse. The public self goes private, in: Narrative Revisited: Telling a Story in the Age of New Media, C. Hoffmann, ed., Amsterdam, Benjamins, pp. 163-184.

[11] A. Fetzer and C. Hofmockel, Signalling discourse relations in context: Degrees of overtness in argumentative and narrative discourse genres, in: Paper Presented at the 3rd Conference on Experimental Approaches to Perception \& Production of Language Variation, Vienna, Austria, 2016.

[12] J.B. Freeman, Dialectics and the Macrostructure of Argument, Foris, Berlin, 1991. doi:10.1515/9783110875843.

[13] T. Givón, English Grammar. A Function-Based Introduction (2 Vols), Benjamins, Amsterdam, 1993.

[14] N. Green, Annotating evidence-based argumentation in biomedical text, in: Proceedings of the IEEE Workshop on Biomedical and Health Informatics, 2015.

[15] M.A.K. Halliday, Introduction to Functional Grammar, 2nd edn, Arnold, London, 1994. 
[16] M. Hannay, The theme zone, in: Nauwe Betrekkingen, R. Boogart and J. Noordegraaf, eds, Nodus Publikationen, Amsterdam, Neerlandistiek and Münster, 1994, pp. 107-117.

[17] C. Hofmockel, A. Fetzer and R.M. Maier, Discourse relations: Genre-specific degrees of overtness in argumentative and narrative discourse, in: Proceedings of the Foundations of the Language of Argumentation at COMMA 2016 Workshop, Potsdam, Germany, 2016, pp. 25-31.

[18] W. Labov, Some further steps in narrative analysis, The Journal of Narrative and Life History 7(1-4) (1997), $395-415$. doi:10.1075/jnlh.7.49som.

[19] S.C. Levinson, Activity types and language, Linguistics 17(5-6) (1979), 365-400. doi:10.1515/ling.1979.17.5-6.365.

[20] N. Madnani, M. Heilman and J. Tetreau, Identifying high-level organizational elements in argumentative discourse, in: Proceedings of the Conference of the North American Chapter of the Association for Computational Linguistics: Human Language Technologies, Montreal, Canada, 2012, pp. 20-28.

[21] R.M. Maier, C. Hofmockel and A. Fetzer, The negotiation of discourse relations in context: Co-constructing degrees of overtness, Intercultural Pragmatics 13(1) (2016), 71-105. doi:10.1515/ip-2016-0003.

[22] W. Mann and S. Thompson, Rhetorical structure theory: Towards a functional theory of text organization, TEXT 8 (1988), 243-281.

[23] D. Marcu, The rhetorical parsing of unrestricted texts: A surface-based approach, Computational Linguistics 26(3) (2000), 395-448. doi:10.1162/089120100561755.

[24] J. Moeschler, Pragmatic connectives, argumentative coherence and relevance, Argumentation 3 (1989), 321-339. doi:10. 1007/BF00128944.

[25] T. Nyan, Metalinguistic Operators. With Reference to French, Peter Lang, Bern, 1998.

[26] A. Peldszus and M. Stede, From argument diagrams to automatic argument mining: A survey, International Journal of Cognitive Informatics and Natural Intelligence (IJCINI) 7(1) (2013), 1-31. doi:10.4018/jcini.2013010101.

[27] A. Peldszus and M. Stede, An annotated corpus of argumentative microtexts, in: Argumentation and Reasoned Action: Proceedings of the 1st European Conference on Argumentation, Lisbon 2015, Vol. 2, College Publications, London, 2016, pp. 801-816.

[28] A. Peldszus and M. Stede, Rhetorical structure and argumentation structure in monologue text, in: Proceedings of the $3 r d$ Workshop on Argumentation Mining, ACL, Berlin, Germany, 2016.

[29] T. Sanders, Semantic and pragmatic sources of coherence: On the categorization of coherence relations in context, Discourse Processes 24(1) (1997), 119-147. doi:10.1080/01638539709545009.

[30] T. Sanders, W. Spooren and L. Noordman, Toward a taxonomy of coherence relations, Discourse Processes 15 (1992), 1-35. doi:10.1080/01638539209544800.

[31] E.M. Segal, J.F. Duchan and P.J. Scott, The role of interclausal connectives in narrative structuring: Evidence from adults' interpretations of simple stories, Discourse Processes 14 (1991), 27-54. doi:10.1080/01638539109544773.

[32] A. Speyer and A. Fetzer, The coding of discourse relations in English and German argumentative discourse, in: The Pragmatics of Discourse Coherence. Theories and Applications, H. Gruber and G. Redeker, eds, Benjamins, Amsterdam, 2014, pp. 87-119.

[33] A. Speyer and A. Fetzer, "Well would you believe it, I have failed the exam again": Discourse relations in English and German personal narratives, Pragmatics and Society (2017), accepted.

[34] M. Stede, S. Afantenos, A. Peldszus, N. Asher and J. Perret, Parallel discourse annotations on a corpus of short texts, in: Proceedings of the International Conference on Language Resources and Evaluation (LREC), Portoroz, Slovenia, 2016.

[35] P. Thibault, Contextualization and social meaning-making practices, in: Language and Interaction: Discussions with John J. Gumperz, S.L. Eerdmans, C.L. Prevignano and P.J. Thibault, eds, John Benjamins, Amsterdam, 2003, pp. 41-62. doi:10. 1075/z.117.05thi.

[36] F.H. van Eemeren, P. Houtlosser and A.F. Snoeck Henkemans, Dialectical profiles and indicators of argumentative moves, Journal of Pragmatics 40 (2008), 475-493. doi:10.1016/j.pragma.2007.12.002. 